\title{
New Zealand National Vegetation Databank
}

\author{
Hazel Broadbent, Nick Spencer \& Susan Wiser
}

\begin{abstract}
The National Vegetation Survey (NVS) databank (GIVD ID AU-NZ-001) is New Zealand's primary archive for plot-based vegetation data and holds data from 77,000 relevés and over 19,000 permanent plots. The NVS databank provides a unique record, spanning more than 50 years, of indigenous and exotic plants in New Zealand's terrestrial ecosystems. A broad range of habitats are covered, with special emphasis on indigenous forests and grasslands. The databank is both an electronic database and physical archive, which stores field recorded plot sheets, maps, and photographs from over 1,200 vegetation surveys. Data in the NVS databank has been built up over many decades by contributions from vegetation scientists and New Zealand environmental conservation agencies. The principal goals of the NVS databank are to provide a secure repository for such data and to ensure quality data are readily available to end-users in different organisations. A website (http://nvs.landcareresearch.co.nz) provides general background information, protocols for data deposit and use, and the ability to conduct online searches of metadata and to request data. Data within NVS have been used to support reporting requirements for the Convention on Biological Diversity, Framework Convention on Climate Change, NZ Resource Management Act, and the Montreal Process. They also assist in ecological restoration, and have been significant in enabling New Zealand to address issues of current concern that were unforeseen at the time of data collection. These include assessing the impacts of climate change and carbon storage in indigenous ecosystems. In 2007 a new extended and robust data model, based in part on the US VegBank design, was built and in 2009 a freely available data entry and analysis tool for vegetation plot data stored within the databank called 'NVS Express' was released. Future goals are to improve Internet services, develop additional online analysis and mapping capabilities, improve integration with plant trait data, and support the Veg-X data exchange schema.
\end{abstract}

Keywords: archive; electronic; grassland; indigenous forest; New Zealand; physical; plot; Veg-X.

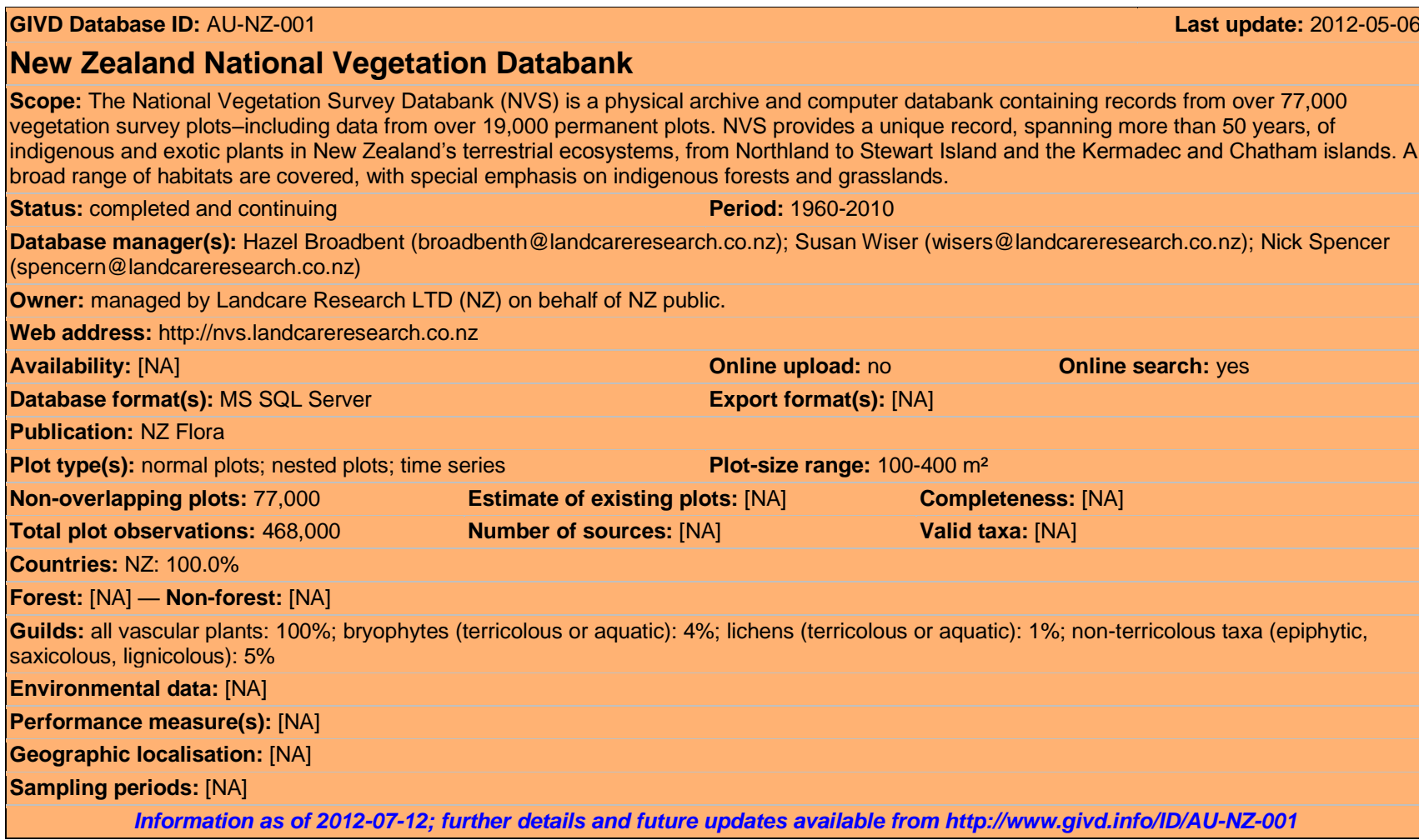

Hazel Broadbent* (broadbenth@landcareresearch.co.nz), Susan Wiser (wisers@ landcareresearch.co.nz), Nick Spencer (spencern@landcareresearch.co.nz)Landcare Research LTD (NZ), Gerald, 7640 Lincoln, NEW ZEALAND

*Corresponding author 